\title{
Novel feature selection method via kernel tensor decomposition for improved multi-omics data analysis
}

\author{
Y.-H. Taguchi ${ }^{1, *}$ and Turki Turki ${ }^{2 *}$ \\ ${ }^{1}$ Department of Physics, Chuo University, Tokyo 112-8551, Japan and ${ }^{2}$ King Abdulaziz University, Department of \\ Computer Science, Jeddah 21589, Saudi Arabia
}

Received YYYY-MM-DD; Revised YYYY-MM-DD; Accepted YYYY-MM-DD

\begin{abstract}
Feature selection of multi-omics data analysis remains challenging owing to the size of omics datasets, comprising approximately $10^{2}-10^{5}$ features. In particular, appropriate methods to weight individual omics datasets are unclear, and the approach adopted has substantial consequences for feature selection. In this study, we extended a recently proposed kernel tensor decomposition (KTD)-based unsupervised feature extraction (FE) method to integrate multi-omics datasets obtained from common samples in a weight-free manner.

KTD-based unsupervised FE was reformatted as the collection of kernelized tensors sharing common samples, which was applied to synthetic and real datasets. The proposed advanced KTD-based unsupervised FE method showed comparative performance to that of the previously proposed KTD method, as well as tensor decompositionbased unsupervised $\mathrm{FE}$, but required reduced memory and central processing unit time. Moreover, this advanced KTD method, specifically designed for multi-omics analysis, attributes $P$-values to features, which is rare for existing multi-omicsoriented methods. The sample $R$ code is available at https://github.com/tagtag/MultiR/
\end{abstract}

\section{INTRODUCTION}

Feature selection with multi-omics datasets has been a longstanding challenge for bioinformatics. Among the numerous proposed methods adapted to multi-omics data analysis $(1,2)$, only few are capable of performing feature selection. Most of these methods fail to implement feature selection because multi-omics data analysis has a strong tendency to involve a small number $(=n)$ of samples with a large number $(=p)$ of features, commonly referred to as the large $p$ small $n$ problem (3), posing difficulty for accurate feature selection. Features should have a sufficiently small $P$-value to be selected under the null hypothesis. Since the raw $P$-values must be heavily corrected for multiple comparisons when dealing with multi-omics datasets, $P$-values become larger and inevitably less significant; thus, attributing significant $P$ values to individual features, even after correction, is difficult. However, since the number of samples (i.e., conditions) is less than that of features (i.e., variables), labels or values attributed to samples can be accurately predicted by any model (when the number of conditions is less than the number of variables, either the labels or values attributed to samples may be predicted, even if the variables are purely random numbers).

In multi-omics analysis, it is difficult to obtain large sample sizes since multiple observations, each of which corresponds to the individual omics approaches, must be performed. In this sense, the required cost and time are multiplied in proportion to the number of omics approaches considered. This often results in a smaller number of samples to which multi-omics measurements are performed when only limited experimental resources are available.

Principal component analysis (PCA) and tensor decomposition (TD)-based unsupervised feature extraction (FE) (4) have been proposed to be applied to feature selection for addressing the large $p$ small $n$ problem. Thus, these approaches are also suitable to feature selection in multiomics analysis. Recently, the TD-based method was extended to kernel TD (KTD)-based unsupervised FE (5), which was applied to integrated analysis of N6-methyladenosine (m6A) and gene expression data (6). These methods attribute $P$-values to features, which is critically important since this enables evaluation of the significance of the selected features, which is rarely possible using other methods applicable to multi-omics datasets $(1,2)$. In spite of this advantage, there are limitations of PCA, TD, and KTD-based unsupervised FE when applied to multi-omics data analysis. We here describe a modification of the KTD-based unsupervised FE method to be more suitable for multi-omics data analysis. Although only a small modification was implemented, it nevertheless resulted in more flexibility for multi-omics data analysis, which was verified using synthetic and real data.

\footnotetext{
*To whom correspondence should be addressed. Tel: +81 3 38171891; Fax: +81 3 38172792; Email: tag@ granular.com
} 


$$
x_{j_{1} \cdots j_{m} j_{1}^{\prime} \cdots j_{m}^{\prime} k}=\sum_{\ell_{1}=1}^{M_{1}} \cdots \sum_{\ell_{m}=1}^{M_{m}} \sum_{\ell_{m+1}=1}^{M_{1}} \cdots \sum_{\ell_{2 m}=1}^{M_{m}} \sum_{\ell_{2 m+1}=1}^{K} G\left(\ell_{1}, \cdots, \ell_{m}, \ell_{m+1}, \cdots, \ell_{2 m}, \ell_{2 m+1}\right)\left(\prod_{s=1}^{m} u_{\ell_{s} j_{s}}\right)\left(\prod_{s^{\prime}=1}^{m} u_{\ell_{m+s^{\prime}} j_{s^{\prime}}^{\prime}}\right) u_{\ell_{2 m+1} k},
$$

\section{MATERIALS AND METHODS}

\section{Extended KTD-based unsupervised FE method}

Suppose that we have $K$ multi-omics datasets with $N_{k}$ features formatted as tensors sharing sample indices $j_{1}, \cdots, j_{m}$ as:

$x_{i_{k} j_{1} j_{2} \cdots j_{m}} \in \mathbb{R}^{N_{k} \times M_{1} \times M_{2} \times \cdots \times M_{m}}$.

$j_{s},\left(1 \leq j_{s} \leq M_{s}\right)$ refers to the $j_{s}$ th measurement in the sth experiment type. $M_{s},(1 \leq s \leq m)$ is the number of measurements in the $s$ th experiment type. Typical examples of $m$ experimental conditions include human subjects, tissues, and time points. For example, if the measurements are performed for $M_{2}$ tissue types from $M_{1}$ individuals at $M_{3}$ time points, the total number of samples is $M_{1} \times M_{2} \times M_{3}$. $i_{k},\left(1 \leq i_{k} \leq N_{k}\right)$ refers to the $i_{k}$ th feature of the $k$ th omics dataset. When $K$ types of omics data are measured for each sample, $k \in[1, K]$.

$x_{i_{k} j_{1} j_{2} \cdots j_{m}}$ can be kernelized as

$$
\begin{aligned}
x_{j_{1} \cdots j_{m} j_{1}^{\prime} \cdots j_{m}^{\prime} k} & =K^{k}\left(x_{i_{k} j_{1} j_{2} \cdots j_{m}}, x_{i_{k} j_{1}^{\prime} j_{2}^{\prime} \cdots j_{m}^{\prime}}\right) \\
& \in \mathbb{R}^{M_{1} \times \cdots \times M_{m} \times M_{1} \times \cdots \times M_{m} \times K},
\end{aligned}
$$

where $K^{k}$ is an arbitrary kernel applied to $x_{i_{k} j_{1} j_{2} \cdots j_{m}}$. Higher-order singular-value decomposition (HOSVD) (4) is then applied to $x_{j_{1} \cdots j_{m} j_{1}^{\prime} \cdots j_{m}^{\prime} k}$, resulting in eq. (4), where $\ell_{s},(1 \leq s \leq 2 m)$ refers to the $\ell_{s}$ th singular-value vectors attributed to the $s$ th experiment type for $1 \leq s \leq m$ and the $(s-2 m)$ th experiment type for $m+1 \leq s \leq 2 m$, respectively. $\ell_{2 m+1}$ refers to the $\ell_{2 m+1}$ th singular-value vector attributed to the omics datasets, $k . u_{\ell_{s} j_{s}} \in \mathbb{R}^{M_{s} \times M_{s}}$ and $u_{\ell_{2 m+1} k} \in$ $\mathbb{R}^{K \times K}$ are singular-value matrices, which are also orthogonal matrices,

$$
\begin{aligned}
\sum_{j_{s}=1}^{M_{s}} u_{\ell_{s} j_{s}} u_{\ell_{s}^{\prime} j_{s}} & =\delta_{\ell_{s} \ell_{s}^{\prime}} \\
\sum_{\ell_{s}=1}^{M_{s}} u_{\ell_{s} j_{s}} u_{\ell_{s} j_{s}^{\prime}} & =\delta_{j_{s} j_{s}^{\prime}} \\
\sum_{k=1}^{K} u_{\ell_{2 m+1} k} u_{\ell_{2 m+1}^{\prime} k} & =\delta_{\ell_{2 m+1} \ell_{2 m+1}^{\prime}} \\
\sum_{\ell_{2 m+1}=1}^{K} u_{\ell_{2 m+1} k} u_{\ell_{2 m+1} k^{\prime}} & =\delta_{k k^{\prime}},
\end{aligned}
$$

where $\delta_{\ell_{s} \ell_{s}^{\prime}}, \delta_{j_{s} j_{s}^{\prime}}, \delta_{\ell_{2 m+1} \ell_{2 m+1}^{\prime}}, \delta_{k k^{\prime}}$ are Kronecker's delta. Because of symmetry, $u_{\ell_{s} j_{s}}=u_{\ell_{s+2 m} j_{s}}$.
$G\left(\ell_{1}, \cdots, \ell_{m}, \ell_{m+1}, \cdots, \ell_{2 m}, \ell_{2 m+1}\right) \in$ $\mathbb{R}^{M_{1} \times \cdots \times M_{m} \times M_{1} \times \cdots \times M_{m} \times K}$ is a core tensor that represents the weight of individual terms composed of the products of singular-value vectors. Here, one should note that $u_{\ell_{2 m+1} k}$ represents the balance (weight) between multi-omics datasets, which usually must be pre-defined manually in the case of a conventional supervised learning approach for FE.

Next, the $u_{\ell_{s} j_{s}}$ values that are of interest from the biological point of view (e.g., distinct values between two classes being compared) must be identified. Using these selected $u_{\ell_{s} j_{s}}$ values, singular-value vectors are derived and assigned to $i_{k} \mathrm{~s}$ as

$u_{i_{k}}^{\ell}=\sum_{j_{1}=1}^{M_{1}} \cdots \sum_{j_{m}=1}^{M_{m}} x_{i_{k} j_{1} j_{2} \cdots j_{m}}\left(\prod_{s=1}^{m} u_{\ell_{s} j_{s}}\right)$

where $\ell=\left(\ell_{1}, \cdots, \ell_{m}\right)$.

Finally, $P$-values are attributed to $i_{k}$ assuming that the $u_{i_{k}}^{\ell}$ values obey a multivariate Gaussian distribution (null hypothesis) as

$P_{i_{k}}=P_{\chi^{2}}\left[>\sum_{\ell}\left(\frac{u_{i_{k}}^{\ell}}{\sigma_{\ell}}\right)^{2}\right]$

where $P_{\chi^{2}}[>x]$ is a cumulative $\chi^{2}$ distribution in which the argument is larger than $x$ and $\sigma_{\ell}$ is the standard deviation. Here, summation is taken over $\ell_{s}$ selected as being of interest. $P$-values are then computed by the pchisq function in R (7).

The obtained $P_{i_{k}}$ values are corrected using the BenjaminiHochberg (BH) criterion (4) and the $i_{k}$ values associated with the adjusted $P$-values less than the established threshold (typically 0.01) are selected. Correction by the $\mathrm{BH}$ criterion is performed by the $\mathrm{p}$. adjust function in $\mathrm{R}$ with the option of method="BH."

\section{Synthetic dataset}

A synthetic dataset was derived in the form of a tensor, $x_{i j k} \in$ $\mathbb{R}^{N \times M \times K}$, as

$x_{i j k}=\left\{\begin{array}{cc}\epsilon_{i j k}+a_{j} i \leq N_{1}, k N_{1}<i \leq(k+1) N_{1} \\ \epsilon_{i j k}\end{array}\right.$,

where

$a_{j}=1+\frac{(M-1) j_{1}}{M}$

and $\epsilon_{i j k} \in(0,1)$ is a uniform random real number that emulates residuals. $N$ is the number of variables, $j$ is a 
variable that adds order to the $j$ th sample, and $k$ is the $k$ th omics data. This synthetic dataset assumes that only the top $N_{1}$ features among $N$ features have dependency on $i$ independent of $k . x_{i j k},\left(k N_{1}<i \leq(k+1) N_{1}\right)$ also has dependence on $j$, but in an omics $(k)$-dependent manner. The dependence on $j$ is a linear increase upon $j$.

\section{Multi-omics hepatitis B virus (HBV) vaccine dataset}

The real multi-omics HPV vaccine datasets were based on 75 samples measured in 15 individuals at five subsequent time points (i.e., 0, 1, 3, 7, and 14 days) after HBV vaccine treatment. Gene expression was measured using RNA-sequencing technology and methylation profiles were measured using microarray technology. The proteome was measured for whole blood cells (WBCs) as well as plasma. Since this multi-omics dataset is composed of four types of omics data measured for 15 individuals at five time points it is thus formatted as a tensor.

Gene expression and methylation profiles were retrieved from the Gene Expression Omnibus (GEO) database using the GSE155198 and GSE161020 datasets, respectively. For gene expression profiles, the GSE155198_RAW.tar dataset was available in the Supplementary File section of GEO. Individual files that included count number of mapped reads toward genes were collected and integrated as a single file. Individual files are named according to the format "GSM $X X X X X X X{ }_{-}$GR $n n_{-}$Vm.count.txt.gz," where $X X X X X X X, n n$, and $m$ are integers; $n n \in$ $\{01,02,03,04,05,06,07,10,11,13,15,17,18,19\}$ identifies the 15 individuals; and $m \in\{3,4,5,6,7\}$ identifies the five time points. Files were loaded into $\mathrm{R}$ as a data frame using the read. CSV command. Data frames were bound into a single data frame using the cbind command in R. For the methylation profiles, the GSE161020_series_matrix.txt.gz dataset, available in the GEO Series Matrix File(s) section, was used as is. The file was loaded into $R$ as a data frame using the read. CSV command. The first column of the data frame is an identifier in the form of cgyyyyyyyy, where yyyyyyyy is an integer. Since the methylation profile was measured with microarray technology, the identifier can be annotated with the reference to the microarray annotation file, GPL6480-9577.txt.gz, which is available under GEO ID GPL6480. Since the other 75 column names are in the form of GSM $X X X X X X X$, the columns were reordered with reference to the columns of the data frame generated from the gene expression profiles as described above. The proteome dataset was obtained from ProteomeXchange (8) using ID PXD020474. Two files (GR01,04,09,10,11,13,15,17,18,19.txt and GR02,03,05,06,07.txt) were downloaded and loaded as data frames into $\mathrm{R}$ using the read. CSV command. The fourth column of the data frame includes the protein IDs, which were used as the identifiers for subsequent analysis. These two data frames were merged with the row names of the union of the protein identifier. Missing observations were filled with zeros. Since the first and second rows have the format "GR $n n$ " and "Visit $m$," respectively, the columns can also be reordered with reference to the column names of the data frame generated by gene expression profiles as described above. $x_{i_{k} j_{1} j_{2}} \in \mathbb{R}^{N_{k} \times 5 \times 15}$; that is, the number of values of the number of $i_{k}$ features of the $k$ th feature type measured at the $j_{1}$ th time point for $j_{2}$ individuals. These values are standardized as $\sum_{i_{k}=1}^{N_{k}} x_{i_{k} j_{1} j_{2}}=0$ and $\sum_{i_{k}=1}^{N_{k}} x_{i_{k} j_{1} j_{2}}^{2}=$ $N_{k} ; N_{k}=687582(k=1$ : methylation $), 35829(k=2:$ gene expression), 1588 ( $k=3$ : WBC proteome and $k=4$ : plasma proteome). Figure 1 schematically illustrates the analysis method for the HBV vaccination datasets.

\section{Kidney cancer multi-omics datasets}

Full description of compilation of the kidney cancer multiomics datasets is available in the related study (9). In brief, there were two sets of multi-omics kidney cancer data, each of which was composed of messenger RNA (mRNA) and microRNA (miRNA) expression profiles. The first dataset was obtained from The Cancer Genome Atlas (TCGA) and included 253 kidney tumors and 71 normal kidneys. The second dataset was obtained from GEO (GSE16441), and included 17 patients and 17 healthy controls. The method by which these two datasets were pre-processed is described in (9). The dataset was formatted as $x_{i_{k} j} \in \mathbb{R}^{N_{k} \times M}$, which represents The $i_{k}$ expression levels of $j$ subjects $(k=1$ for mRNA and $k=2$ for miRNA).

\section{RESULTS}

\section{Synthetic dataset}

One hundred ensembles of $x_{i j k}$ were generated and the performances were averaged. First, a linear kernel was generated as

$x_{k j j^{\prime}}=K^{k}\left(x_{i j k}, x_{i_{k} j^{\prime} k}\right)=\sum_{i=1}^{N} x_{i j k} x_{i j^{\prime} k} \in \mathbb{R}^{M \times M \times K}$.

Then, HOSVD was applied, resulting in

$x_{k j j^{\prime}}=\sum_{\ell_{1}=1}^{M} \sum_{\ell_{2}=1}^{M} \sum_{\ell_{3}=1}^{K} G\left(\ell_{1} \ell_{2} \ell_{3}\right) u_{\ell_{1} j} u_{\ell_{2} j^{\prime}} u_{\ell_{3} k}$.

(for the dimensions of the datasets, $N, M$, and $K$, see the legend to Fig. 1).Since it was observed that $u_{2 j}$ always had the largest correlation with $a_{j}$ and $u_{1 k}$ was always constant, regardless of $k, u_{2 j}$ and $u_{1 k}$ were employed to compute

$u_{\ell_{1} \ell_{2} i}=\sum_{j_{1}=1}^{M} \sum_{k=1}^{3} x_{i j k} u_{\ell_{j}} u_{\ell_{2} k}$

for attributing the $P$-values

$P_{i}=P_{\chi^{2}}\left[>\left(\frac{u_{\ell_{1} \ell_{2} i}}{\sigma_{\ell_{1} \ell_{2}}}\right)^{2}\right]$

The $P_{i}$ values were corrected using the $\mathrm{BH}$ criterion and the $i$ values associated with adjusted $P_{i}$ less than 0.01 were 


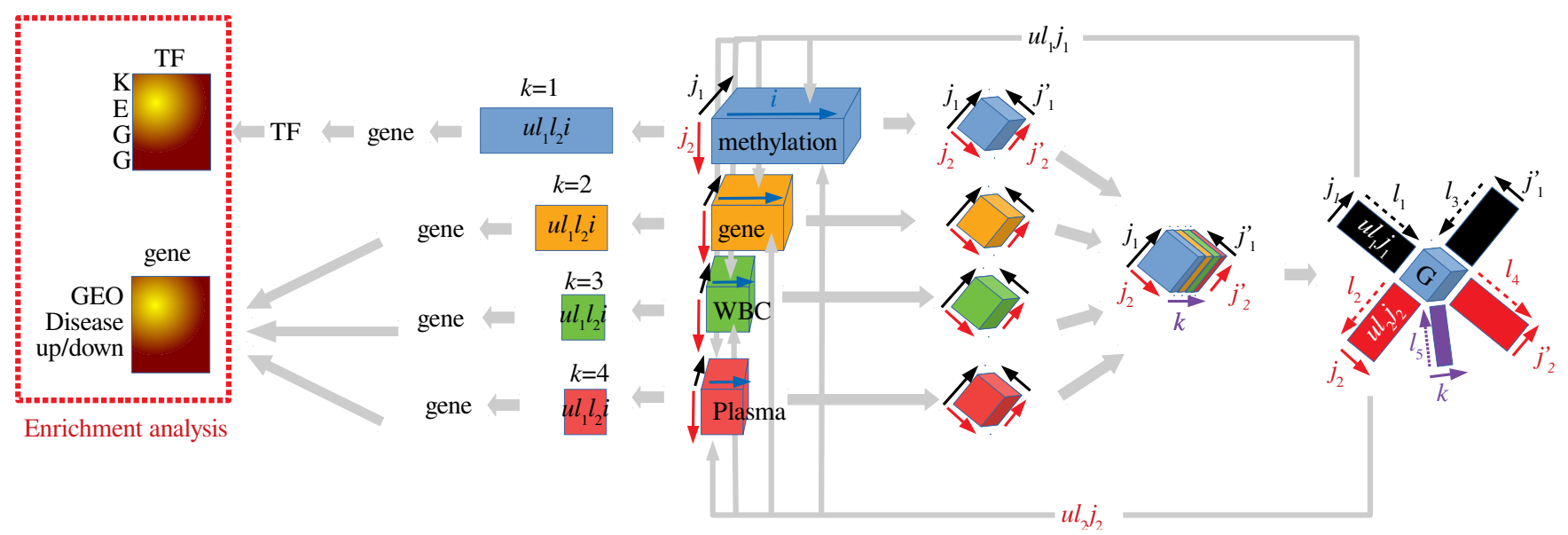

Figure 1. Schematic representation of hepatitis B virus (HBV) vaccination data analysis. Analysis starts from the center, moves to the right, comes back to the center, and then moves to the left. The cyan rectangle annotated as "methylation" is $x_{i_{1} j_{1} j_{2} 1}$, the yellow rectangle annotated as "gene" is $x_{i_{2}} j_{1} j_{2} 2$, the green rectangle annotated as "WBC" is $x_{i_{3} j_{1} j_{2} 3}$, and the magenta rectangle annotated as "Plasma" is $x_{i_{4} j_{1} j_{2} 4}$. The four tilted cubes to the right of these four rectangles are $x_{j_{1} j_{2} j_{1}^{\prime} j_{2}^{\prime} k}$, whose correspondence with $x_{i_{k} j_{1} j_{2} k}$ is indicated by the same color. The tilted cubes colored by layers to the right of the four tilted cubes represent the bundle of $x_{j_{1} j_{2} j_{1}^{\prime} j_{2}^{\prime} k}$. The right-most figure with a blue cube annotated as "G" at the center corresponds to TD shown in eq. (20). The four colored rectangles to the left of the four colored and annotated rectangles represent the singular-value vectors computed by eq. (21). Genes are selected from these singular-value vectors using $P$-values computed by eq. (22). For methylation, transcription factors (TFs) are further selected by Enricher using the selected genes (Table 4). The selected genes and TFs are then uploaded to Enrichr to validate the biological reliability (the left-most figure with color gradation).

Table 1. Confusion matrix when applying KTD-based unsupervised FE to a synthetic dataset $\left(N=1000, N_{1}=10, M=10, K=3\right)$.

\begin{tabular}{l|cc}
\hline & adjusted $P_{i} \leq 0.01$ & adjusted $P_{i}>0.01$ \\
\hline$i \leq N_{1}$ & 7.06 & 2.94 \\
$i>N_{1}$ & 0.04 & 989.96 \\
\hline
\end{tabular}

selected. Table 1 shows the confusion matrix. Among the 10 features associated with $a_{i}$, approximately seven features were correctly selected, whereas false positives were almost zero. Thus, KTD-based unsupervised FE successfully selected features correlated with $a_{j}$.

To demonstrate the difficulty of this task, linear regression was used as an alternative method

$x_{i j k}=\alpha_{i k} a_{j}+\beta_{i k}$,

where $\alpha_{i k}$ and $\beta_{i k}$ are regression coefficients, and $a_{j}$ is defined in eq. (12). Since $x_{i j k} \mathrm{~s}$ with distinct $k$ differ, distinct models were applied to each. After computing BH-corrected $P$ values, the $i$ s associated with adjusted $P$-values less than 0.01 were selected. Table 2 shows the confusion matrix. Essentially, no features correlated with $a_{j}$ were selected. Thus, regression analysis did not select any features correlated with $a_{j}$.

These results demonstrated that an apparently simple and easy problem became difficult when it is a large $p$ small $n$ problem, whereas KTD-based unsupervised FE was able to handle this problem to some extent. These advantages have also been observed in PCA- and TD-based unsupervised FE (4).

Although the methods that did not attribute $P$-values to features were not of interest, since the capability of attributing
Table 2. Confusion matrix when linear regression was applied to the synthetic dataset $\left(N=1000, N_{1}=10, M=10, K=3\right.$ ).

\begin{tabular}{c|cc}
\hline & $\begin{array}{c}\text { adjusted } \\
P_{i} \leq 0.01\end{array}$ & $\begin{array}{c}\text { adjusted } \\
P_{i}>0.01\end{array}$ \\
\hline$k=1$ & 0.07 & 19.93 \\
$i \leq 2 N_{1}$ & 0.03 & 979.97 \\
$i>2 N_{1}$ & & \\
$k=2$ & 0.07 & 19.93 \\
$i \leq N_{1}, 2 N_{1}<i \leq 3 N_{1}$ & 0.01 & 979.99 \\
Other than above & & \\
$k=3$ & & 19.91 \\
$i \leq N_{1}, 3 N_{1}<i \leq 4 N_{1}$ & 0.09 & 979.99 \\
Other than above & 0.01 & \\
\hline
\end{tabular}

$P$-values to features is a great advantage of KTD-based unsupervised FE, as emphasized in the Introduction, least absolute shrinkage and selection operator (lasso) (10) was employed as another method for comparison. Although lasso regression does not attribute $P$-values to features, the model was fitted to feature selection in a large $p$ small $n$ problem to demonstrate the difficulty of feature selection in the synthetic dataset. Table 3 shows the confusion matrix, which is obviously inferior to that shown in Table 1. Although the KTD-based unsupervised FE approach correctly selected at least 7 out of 10 features $(70 \%)$, which was correlated with $a_{j}$ with essentially no false positives, lasso selected at most 5 out of 20 features (only $25 \%$ ), which was correlated with $a_{j}$ with two false positives (approximately half of the true positives). This suggested that even if methods that could not attribute $P$-values to features can be considered, they would not outperform the KTD-based unsupervised FE method. Thus, subsequently, we only focused only methods that attributed $P$-values to features. 
Table 3. Confusion matrix when least absolute shrinkage and selection operator (lasso) was applied to the synthetic dataset $\left(N=1000, N_{1}=\right.$ $10, M=10, K=3)$.

\begin{tabular}{c|cc}
\hline & $\begin{array}{c}\text { adjusted } \\
P_{i} \leq 0.01\end{array}$ & $\begin{array}{c}\text { adjusted } \\
P_{i}>0.01\end{array}$ \\
\hline$k=1$ & 4.62 & 15.383 \\
$i \leq 2 N_{1}$ & 2.12 & 977.88 \\
$i>2 N_{1}$ & & \\
$k=2$ & 4.70 & 15.30 \\
$i \leq N_{1}, 2 N_{1}<i \leq 3 N_{1}$ & 2.27 & 977.73 \\
Other than the above & & \\
$k=3$ & 4.55 & 15.45 \\
$i \leq N_{1}, 3 N_{1}<i \leq 4 N_{1}$ & 2.12 & 977.78 \\
Other than the above & & \\
\hline
\end{tabular}

\section{HBV vaccine dataset}

A linear kernel was employed as follows:

$K^{k}\left(x_{i_{k} j_{1} j_{2}}, x_{i_{k} j_{1}^{\prime} j_{2}^{\prime}}\right)=\sum_{i_{k}=1}^{N_{k}} x_{i_{k} j_{1} j_{2}} x_{i_{k} j_{1}^{\prime} j_{2}^{\prime}}$

Then, a tensor was added:

$x_{k j_{1} j_{2} j_{1}^{\prime} j_{2}^{\prime}}=K^{k}\left(x_{i_{k} j_{1} j_{2}}, x_{i_{k} j_{1}^{\prime} j_{2}^{\prime}}\right) \in \mathbb{R}^{4 \times 5 \times 15 \times 5 \times 15}$.

Applying HOSVD to $x_{k j_{1} j_{2} j_{1}^{\prime} j_{2}^{\prime}}$ results in

$x_{k j_{1} j_{2} j_{1}^{\prime} j_{2}^{\prime}}=\sum_{k=1}^{4} G\left(\ell_{1} \ell_{2} \ell_{3} \ell_{4} \ell_{5}\right) u_{\ell_{1} j_{1}} u_{\ell_{2} j_{2}} u_{\ell_{3} j_{1}^{\prime}} u_{\ell_{4} j_{2}^{\prime}} u_{\ell_{5} k}$

where $\quad G \in \mathbb{R}^{5 \times 15 \times 5 \times 15 \times 4}, \quad u_{\ell_{1} j_{1}}, u_{\ell_{3} j_{1}^{\prime}} \in \mathbb{R}^{5 \times 5}$, $u_{\ell_{2} j_{4}}, u_{\ell_{4} j_{2}^{\prime}} \in \mathbb{R}^{15 \times 15}$, and $u_{\ell_{5} k} \in \mathbb{R}^{4 \times 4}$. Since $x_{k j_{1} j_{2} j_{1}^{\prime} j_{2}^{\prime}}=$ $x_{k j_{1}^{\prime} j_{2} j_{1} j_{2}^{\prime}}$ and $x_{k j_{1} j_{2} j_{1}^{\prime} j_{2}^{\prime}}=x_{k j_{1} j_{2}^{\prime} j_{1}^{\prime} j_{2}}$ because of symmetry, $G\left(\ell_{1} \ell_{2} \ell_{3} \ell_{4} \ell_{5}\right)=G\left(\ell_{1} \ell_{4} \ell_{3} \ell_{2} \ell_{5}\right)=G\left(\ell_{3} \ell_{2} \ell_{1} \ell_{4} \ell_{5}\right)$, $\left\{u_{\ell_{1} j_{1}}\right\}=\left\{u_{\ell_{3} j_{1}^{\prime}}\right\}$, and $\left\{u_{\ell_{2} j_{2}}\right\}=\left\{u_{\ell_{4} j_{2}^{\prime}}\right\}$.

The singular-value vectors of interest were as follows:

- $u_{\ell_{1} j_{1}}$ and $u_{\ell_{3} j_{1}^{\prime}}$ should be significantly dependent on time points corresponding to $j_{1}$ and $j_{1}^{\prime}$.

- $u_{\ell_{2} j_{2}}$ and $u_{\ell_{4} j_{2}^{\prime}}$ should be independent of individuals corresponding to $j_{2}$ and $j_{2}^{\prime}$.

- $u_{\ell_{5} k}$ should be common between distinct omics measurements.

As a result, $\ell_{1}=\ell_{3}=2, \ell_{2}=\ell_{4}=1, \ell_{5}=1$ satisfies the required conditions (Fig. 2). Then,
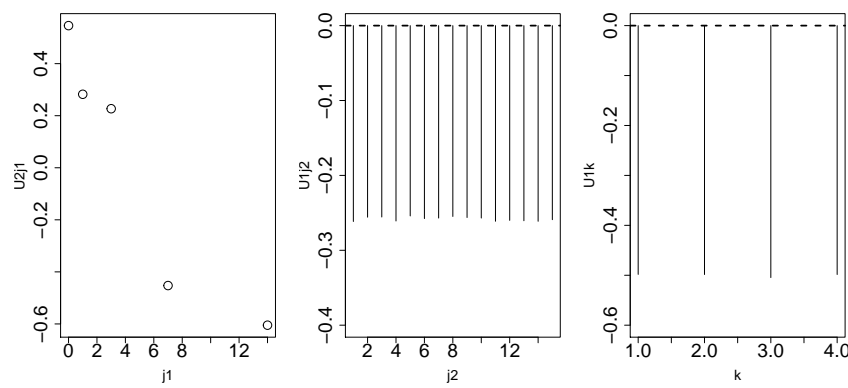

Figure 2. Left: $u_{2 j_{1}}$, middle: $u_{1 j_{1}}$, right: $u_{1 k}$ when HOSVD is applied to a linear kernel computed using hepatitis $B$ virus (HBV) vaccine data. The Pearson correlation coefficient between $j_{1}$ and $u_{2 j_{1}}$ is $-0.94(P=0.02)$.

$u_{i_{k}}=\sum_{j_{1}=1}^{5} \sum_{j_{2}=1}^{15} x_{i_{k} j_{1} j_{2}} u_{2 j_{1}} u_{1 j_{2}}$

is computed and

$P_{i_{k}}=P_{\chi^{2}}\left[>\left(\frac{u_{i_{k}}}{\sigma_{21}}\right)^{2}\right]$

The computed $P_{i_{k}}$ values were corrected using the $\mathrm{BH}$ criterion and $i_{k} \mathrm{~s}$ associated with either $P_{i_{k}}<0.01$ (for gene expression and methylation) or with $P_{i_{k}}<0.05$ (for the two proteomes) were successfully selected (the full lists of selected features are available in Supplementary Data S1-S4).

To validate genes selected by KTD-based unsupervised FE, the selected genes were uploaded to Enrcihr (11). Initially, 1335 genes associated with 2077 methylation probes selected by KTD-based unsupervised FE were uploaded. Many transcription factors (TFs) were significantly predicted to target these 1335 genes (Table 4). These 21 TFs were then uploaded to Enrichr again; Table S1 shows the top 10 ranked Kyoto Encyclopedia of Genes and Genomes (KEGG) pathways in the "KEGG 2019 HUMAN" Enrichr category (the full list is available in Supplementary Data S1). It was clear that these data included many biologically reasonable KEGG pathways (for details, see the Discussion section below).

Table 4. Transcription factors (TFs) enriched in the "ChEA 2016" Enrichr category (adjusted $P$-values $<0.05$ ) when 1335 genes associated with 2077 methylation probes selected by KTD-based unsupervised FE were considered (the full list is available in Supplementary Data S1).

\begin{tabular}{l|l} 
TFs & ZNF217, TCF4, STAT3, SMARCD1, WT1, \\
FOXA2, PAX3-FKHR, SMAD4, SMAD3, SOX9, \\
TFAP2C, YAP1, AR, SOX2, CTNNB1, VDR, \\
PIAS1, TEAD4, MITF, HNF4A, SUZ12
\end{tabular}

Eight genes associated with 11 probes were identified as differentially expressed genes (DEGs) when gene expression profiles were considered (Table 5), which were uploaded to Enrichr. Conversion of probe names to gene symbols was performed by the ID converter tool of DAVID (12). 
Table 5. Eight genes associated with 11 probes identified as differentially expressed genes (DEGs) when gene expression profiles were considered.

Gene symbols | S100A9, CD74, hba1, ACTB, HBB, HBA2, MALAT1, COX1

Table S2 shows the top 10 ranked GEO profiles enriched in the Enrichr "Disease Perturbations from GEO up/down" category (the full list is available in Supplementary Data S2).

Although many neurodegenerative profiles not apparently related to the HBV vaccine are listed, the rationalization for their inclusion is provided in the Discussion section.

Finally, two set of proteins identified as DEGs in WBC and plasma profiles (Table 6) were uploaded to Enrichr.

Table 6. Proteins identified as differentially expressed genes (DEGs) when gene expression profiles in the proteome were considered.

\begin{tabular}{|c|c|}
\hline WBC & $\begin{array}{lcr}\text { HIST1H2BJ, } & \text { HIST2H2BF, } & \text { HIST1H2BG, } \\
\text { HIST1H2BB, } & \text { HIST1H2BD, } & \text { ACTG1, } \\
\text { HIST1H2BL, HIST1H2BN, PFN1, HIST1H2BK, }\end{array}$ \\
\hline & $\begin{array}{lcr}\text { HIST3H2BB, } & \text { ACTB, } & \text { HBB, } \\
\text { HIST1H2BA, } & \text { HIST1H2BI, } & \text { HIST1H2BC, } \\
\text { HIST1H2BO, } & \text { HIST2H2BE, } & \text { HIST1H2BM, } \\
\text { HBA1, HIST1H2BF, HIST1H2BE, HIST1H2BH }\end{array}$ \\
\hline $\begin{array}{c}\text { Plasma } \\
\end{array}$ & $\begin{array}{l}\text { FGA, HP, GSN, ALB, FGG, IGLL5, APOA1, } \\
\text { SERPINA1, ORM1, TF, GC, CP, C4A, CSF3R, } \\
\text { A2M, HPX, HRG, A1BG, CFH, APOB, C3, } \\
\text { CLEC14A }\end{array}$ \\
\hline
\end{tabular}

Tables S3 and S4 show the top 10 ranked enriched GEO profiles identified in the Enrichr "Disease Perturbations from GEO up/down" category when proteins for the WBC and plasma sections in Table 6 were uploaded to Enrichr (the full list is available in Supplementary Data S3 and S4). Other than the enriched reasonable hepatitis-related GEO profiles, some neurodegenerative disease-related GEO profiles were also enriched, as shown in Table S3, which are further explored in the Discussion section.

Since there are many enriched biological processes and pathways listed in Tables S1-S4, the genes and proteins selected in this section may not be artifacts, but likely have a true biological basis.

\section{Kidney cancer}

A linear kernel was employed as

$K^{k}\left(x_{i_{k} j}, x_{i_{k} j^{\prime}}\right)=\sum_{i_{k}=1}^{N_{k}} x_{i_{k} j} x_{i_{k} j^{\prime}} \in \mathbb{R}^{N_{k} \times M}$.

For the first dataset (i.e., TCGA data), $M=324$, whereas for the second dataset (i.e., GEO data), $M=37$. Then, a tensor was added

$x_{k j j^{\prime}}=K^{k}\left(x_{i_{k} j}, x_{i_{k} j^{\prime}}\right) \in \mathbb{R}^{2 \times M \times M}$.

Applying HOSVD to $x_{k j j^{\prime}}$ results in

$x_{k j j^{\prime}}=\sum_{k=1}^{2} G\left(\ell_{1} \ell_{2} \ell_{3}\right) u_{\ell_{1} j} u_{\ell_{2} j^{\prime}} u_{\ell_{3} k}$,
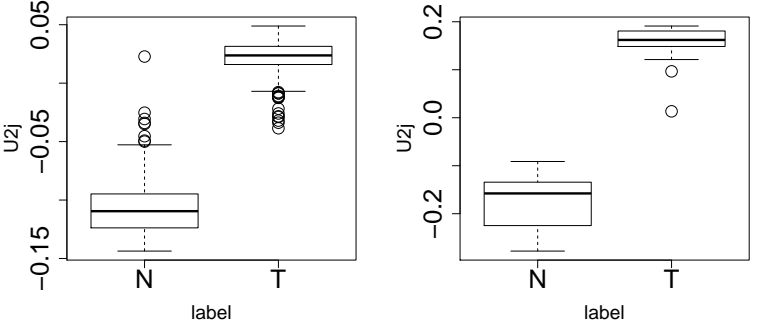

Figure 3. Boxplot of $u_{2 j}$ when higher-order singular-value decomposition (HOSVD) is applied to a linear kernel computed using kidney cancer data. Left: TCGA, $P=8.49 \times 10^{-47}$, right: GEO, $P=4.07 \times 10^{-17} . P$-values are based on the $t$ test applied to $u_{2 j}$. T: tumors, N: normal kidney samples.

where $G \in \mathbb{R}^{M \times M \times 2}, u_{\ell_{1} j}, u_{\ell_{2} j^{\prime}} \in \mathbb{R}^{M \times M}$, and $u_{\ell_{3} k} \in \mathbb{R}^{2 \times 2}$. Since $x_{k j j^{\prime}}=x_{k j^{\prime} j}$ because of symmetry, $G\left(\ell_{1} \ell_{2} \ell_{3}\right)=$ $G\left(\ell_{1} \ell_{3} \ell_{3}\right), \quad\left\{u_{\ell_{1} j}\right\}=\left\{u_{\ell_{2} j^{\prime}}\right\}$. Then, singular-value vectors were identified such that $u_{\ell_{1} j}$ and $u_{\ell_{2} j^{\prime}}$ were significantly distinct between healthy controls and patients. As a result, $\ell_{1}=\ell_{2}=2$ satisfies the required conditions (Fig. 3). Then,

$u_{i_{k}}=\sum_{j=1}^{M} x_{i_{k} j} u_{2 j}$

is computed and

$P_{i_{k}}=P_{\chi^{2}}\left[>\left(\frac{u_{i_{k}}}{\sigma_{2}}\right)^{2}\right]$

The computed $P_{i_{k}}$ values were corrected using the $\mathrm{BH}$ criterion and $i_{k}$ s associated with $P_{i_{k}}<0.01$ were successfully selected. hsa-mir-200c and hsa-mir-141 were selected from TCGA, and hsa-miR-141, hsa-miR-210, and hsa-miR-200c were selected from GEO. Thus, these miRNAs are highly coincident with each other, even more so than reported in previous works $(5,9)$ where TD- as well as KTD-based unsupervised FE was applied to TCGA and GEO datasets. For mRNA, there were five common genes selected between the TCGA and GEO datasets (Table $7, P=6.7 \times 10^{-5}$, odds ratio: 13.13).

Table 7. Confusion matrix of selected mRNAs between The Cancer Genome Atlas (TCGA) and Gene Expression Omnibus (GEO) datasets

\begin{tabular}{lrrr}
\hline & & \multicolumn{2}{c}{ GEO } \\
& & $P>0.01$ & $P<0.01$ \\
\hline TCGA & $P>0.01$ & 17269 & 101 \\
& $P<0.01$ & 65 & 5 \\
\hline
\end{tabular}

$P=6.7 \times 10^{-5}$, Odds ratio: 13.13 
Table 8. Correlation between hepatitis B virus (HBV) vaccine experiment kernels. Upper triangle: Pearson correlation, lower triangle: $P$-values.

\begin{tabular}{llll}
\hline & Gene expression & WBC & Plasma \\
\hline Gene expression & - & 0.1279405 & 0.2192163 \\
WBC & $1.34 \times 10^{-11}$ & - & 0.4384998 \\
Plasma & $1.52 \times 10^{-31}$ & $8.98 \times 10^{-131}$ & - \\
\hline
\end{tabular}

\section{DISCUSSION}

There are several advantages in the proposed implementation of KTD-based unsupervised FE compared with previously proposed versions of KTD-based unsupervised FE (5), as well as TD-based unsupervised FE (4) in the context of application to the integration of multi-omics datasets. When the original implementation of TD-based unsupervised FE (4) is applied to the integration of multi-omics data, (e.g., $x_{i j} \in \mathbb{R}^{N \times M}$ and $x_{h j} \in \mathbb{R}^{H \times M}$, which corresponds to the $i$ th or $h$ th omics data of the $j$ th sample), HOSVD is applied to

$x_{i h}=\sum_{j} x_{i j} x_{h j} \in \mathbb{R}^{N \times H}$.

Since $H, N \gg M$, this was not an effective implementation. When KTD-based unsupervised FE (5) is applied, HOSVD is applied to

$x_{j j^{\prime \prime}}=\sum_{j^{\prime}}\left(\sum_{i} x_{i j} x_{i j^{\prime}}\right)\left(\sum_{h} x_{h j^{\prime}} x_{h j^{\prime \prime}}\right) \in \mathbb{R}^{M \times M}$.

This drastically reduced the required memory and central processing unit (CPU) time. Nevertheless, it was unclear how more than two omics datasets could be integrated. In the implementation introduced in this paper,

$x_{j j^{\prime} k}=\sum_{i} x_{i j k} x_{i j^{\prime} k} \in \mathbb{R}^{M \times M \times K}$,

which corresponds to the $i$ th measurement of the $k$ th omics data of the $j$ th sample, where $K$ is the total number of omics datasets. Since HOSVD is applied to $x_{j j^{\prime} k}$, any number of multi-omics datasets may be handled. This slight modification drastically increased the ability of KTD-based unsupervised FE to handle multi-omics datasets.

Kernels $K^{k}$ were highly correlated between mRNA $(k=$ 1) and miRNA $(k=2)$ for the kidney cancer data (13) (Fig.4). Kernels $K^{k}$ for gene expression profiles and the proteome were also highly correlated when HBV vaccine experiments were considered (Table 8). Thus, it was obvious that the current formalism was very effective in identifying the coincidence between individual omics (in this case, mRNA, miRNA, and the proteome).

Although the list of enriched pathways in Table S1 did not seem to be related to the HBV vaccine directly, there were indirect reasonable relationships. For example, the Hippo signaling pathway has recently been identified to be related to the immune system (14). "Hepatitis B" was also significantly enriched (adjusted $P$-value of $7.79 \times 10^{-3}$ ), but it did not rank
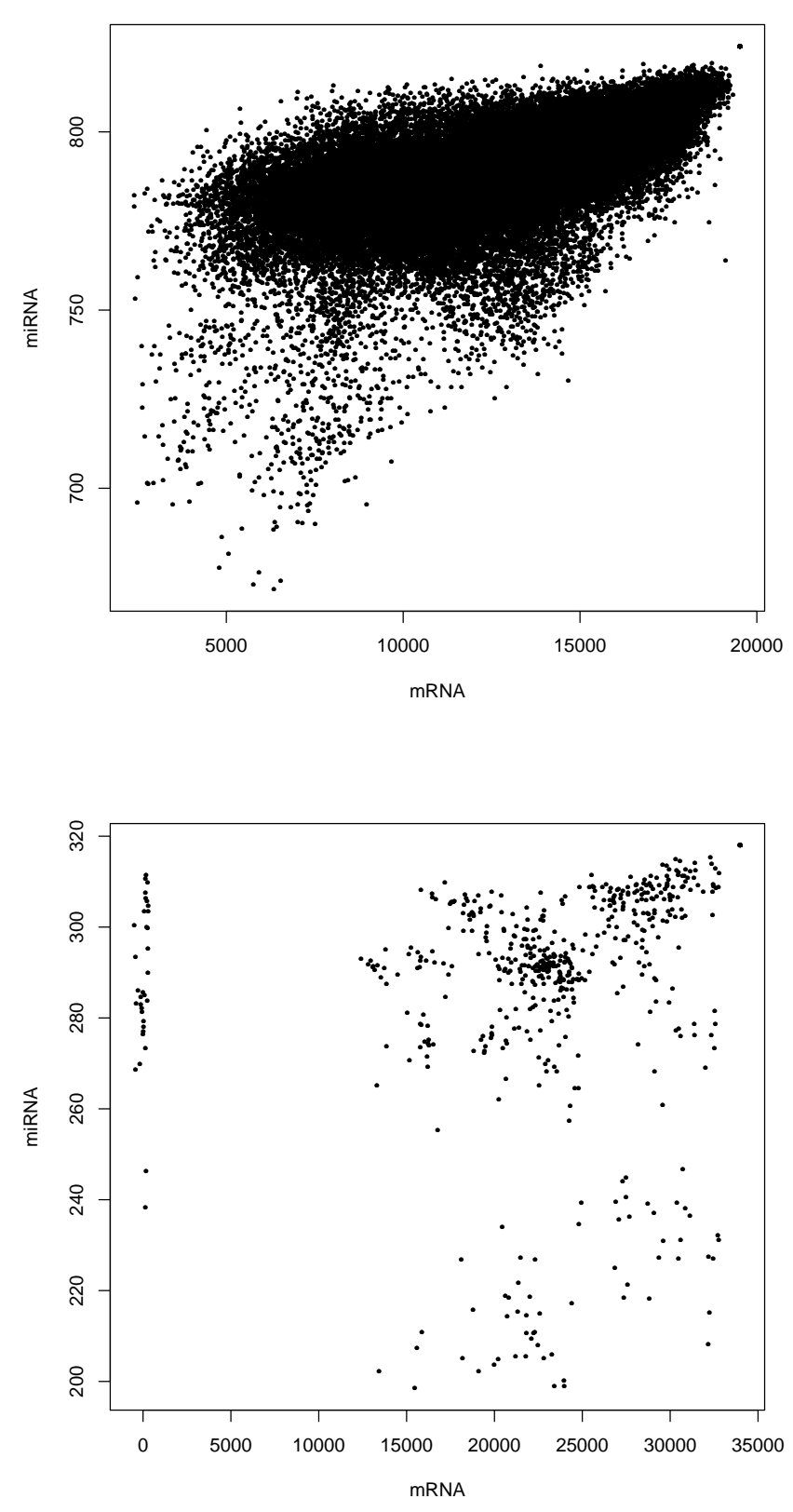

Figure 4. Scatter plot of kernels $K^{k}$ between messenger RNA (mRNA) and microRNA (miRNA). Upper: The Cancer Genome Atlas (TCGA) (Pearson correlation coefficient $=0.627, P=0.00$ ), Lower: Gene Expression Omnibus (GEO)(Pearson correlation coefficient $=0.349, P=2.32 \times 10^{-34}$ ). 
within the top-10 KEGG pathways in Table S1. In addition, since patients with diabetes have a higher risk of HBV infection (15), it is reasonable that the two KEGG pathways "AGE-RAGE signaling pathway in diabetic complications" and "Maturity onset of diabetes in the young" were enriched. Although multiple cancer types were also enriched in these data, as shown in Table S1, many cancer types other than liver cancers are known to be related to risk of HBV infection (16).

Although genes identified as DEGs in relation to $\mathrm{HBV}$ vaccination were also enriched in various neurodegenerative diseases other than hepatitis (Tables S2 and S3), this is a reasonable finding because viral hepatitis was reported to be related to Parkinson's disease (17). There are also known associations between hepatic functions and plasma amyloid$\beta$ levels (18); cirrhosis patients with HBV infection have higher plasma $\mathrm{A} \beta 40$ and $\mathrm{A} \beta 42$ levels than patients with $\mathrm{HBV}$ negative cirrhosis. More directly, Ji et al.(19) reported that the hepatitis B core VLP-based mis-disordered tau vaccine alleviated cognitive deficits and neuropathology progression in a Tau.P301S mouse model of Alzheimer's disease. Thus, enrichment of neurodegenerative disease-related genes among the identified DEGs does not appear to be an artifact, but rather provides possible supportive evidence that KTD-based unsupervised FE detected side effects caused by vaccinations.

Other conventional univariate tools such as limma (20) and sam (21) cannot be used for these tasks since they are designed to handle categorical classes and thus cannot be applied to HBV vaccination data, which are only associated with time points and are not categorical. Although regression analysis was attempted for the synthetic dataset, there were no features correlated with dates. Thus, there were no univariate feature selection methods applicable to the HBV vaccination data that could identify features correlated with date. For the kidney cancer datasets, it has been extensively demonstrated that these conventional univariate tools such as limma (20) and sam (21) cannot compete with the TD-based unsupervised FE approach $(5,9)$. Thus, no univariate feature selection method was identified that was superior to TD-based unsupervised FE when applied to the kidney cancer datasets.

The performance of the proposed method has not been compared to other existing multi-omics-oriented methods (1, 2) because no suitable methods were identified for suitable comparison. First, most of the recently proposed cutting-edge methods adapted to multi-omics analysis are specific to a high-throughput sequencing (HTS) architecture. For example, MKpLMM (22) requires genomic coordinates, which are not available for the datasets analyzed in this study. Similarly, csaw (23) requires a bed file, which is also not available for the present datasets. Since the purpose of the present study was to propose a more flexible method that is not specific to the HTS architecture and the datasets employed in this study were not obtained using HTS, these were considered to be unsuitable methods for comparison with the proposed implementation of KTD-based unsupervised FE. Second, other methods that are not specific to HTS lack the statistical validation of feature selection (i.e., no ability to attribute $P$-values to features). For example, although MOFA (24) is not specific to HTS, it does not have the ability to select features; thus, we were not able to compare its performance with that of our proposed method. Although DIABLO (25) is also not specific to the HTS architecture and has feature selection ability, there is no functionality to attribute $P$-values to individual features; thus, features cannot be selected based on statistical significance, and therefore, DIABLO was considered to be outside of the scope of this study. FSMKL (26), which is also not specific to the HTS architecture does have the ability to add statistical scores to individual features; however, selecting features based on statistical scores is not effective. In this sense, to our knowledge, there are no other multi-omics-oriented feature selection methods that satisfy the following requirements:

- Not specific to the HTS architecture

- Attributes $P$-values to individual features to evaluate statistical significance

- Can handle more than or equal to three kinds of omics data simultaneously

- Applicable to severe large $p$ small $n$ (typically, $p / n \sim$ $10^{2}$ or more) problems

For example, for the large $p$ small $n$ problem, although Subramanian et al.(2) summarized existing machine learning methods for multi-omics analysis, typically they are applied to studies including up to $10^{2}$ samples; thus, they cannot be regarded as a severe large $p$ small $n$ problem. As a result, the performance of the KTD-based unsupervised FE was not directly compared to other existing methods that satisfy all of the above conditions.

HBV vaccination data were selected to demonstrate the superior power of advanced KTD-based unsupervised FE because of the difficulty of the problem with this dataset. Since vaccination must be given to healthy people, side effects must be minimized (27); in fact, since vaccination is essentially an infection with a weaker pathogen, its effect is inevitably weak. As expected, a very limited number of features (genes and proteins) were selected using the advanced KTD-based unsupervised FE proposed in this article, whereas conventional linear regression analysis did not attribute significant $P$-values to any features. This suggests that the proposed advanced KTD-based unsupervised FE method has superior ability to select features when applied to even particularly difficult multi-omics datasets.

\section{CONCLUSION}

In this paper, an advanced KTD-based unsupervised FE method was introduced, which was modified to be applied to feature selection in multi-omics data analysis that is often very difficult, mainly based on the large $p$ small $n$ problem. The proposed method was successfully applied to a synthetic dataset, as well as to two real datasets, and attributed significant $P$-values to features with reduced CPU time and memory, even when applied to integrated analysis of more than two multi-omics datasets. Although the modification from the previously proposed KTD-based unsupervised FE was not significant, this slight modification was successful when applied to feature selection of multi-omics data analysis, which often poses a challenge in the case of a large $p$ small $n$ problem. 


\section{ACKNOWLEDGEMENTS}

This work was supported by Japan Society for the Promotion of Science, KAKENHI [grant numbers 19H05270, 20K12067, 20H04848] to YHT.

Conflict of interest statement. None declared.

\section{LIST OF ABBREVIATION}

BH: Benjamini-Hochberg

DEG: differentially expressed gene

FE: feature extraction

GEO: Gene Expression Omnibus

HBV: hepatitis B virus

HOSVD: higher-order singular-value decomposition

HTS: high-throughput sequencing

KEGG: Kyoto Encyclopedia of Genes and Genomes

KTD: kernel tensor decomposition

PCA: principal component analysis

TCGA: The Cancer Genome Atlas

TD: tensor decomposition

TF: transcription factor

WBC: whole blood cell

\section{REFERENCES}

1. Reel, P. S., Reel, S., Pearson, E., Trucco, E., and Jefferson, E. (2021) Using machine learning approaches for multi-omics data analysis: A review. Biotechnology Advances, 49, 107739.

2. Subramanian, I., Verma, S., Kumar, S., Jere, A., and Anamika, K. (2020) Multi-omics Data Integration, Interpretation, and Its Application. Bioinformatics and Biology Insights, 14, 1177932219899051 PMID: 32076369.

3. Huynh, P.-H., Nguyen, V. H., and Do, T.-N. (June, 2020) Improvements in the Large $\mathrm{p}$, Small $\mathrm{n}$ Classification Issue. SN Computer Science, 1(4).

4. Taguchi, Y.-h. (2020) Unsupervised Feature Extraction Applied to Bioinformatics, Springer International Publishing,

5. Taguchi, Y.-H. and Turki, T. (2021) Mathematical formulation and application of kernel tensor decomposition based unsupervised feature extraction. Knowledge-Based Systems, 217, 106834.

6. Roy, S. S. and Taguchi, Y.-H. (April, 2021) Identification of genes associated with altered gene expression and m6A profiles during hypoxia using tensor decomposition based unsupervised feature extraction. Scientific Reports, 11(1), 8909.

7. R Core Team R: A Language and Environment for Statistical Computing R Foundation for Statistical Computing Vienna, Austria (2020).

8. Deutsch, E. W., Csordas, A., Sun, Z., Jarnuczak, A., Perez-Riverol, Y., Ternent, T., Campbell, D. S., Bernal-Llinares, M., Okuda, S., Kawano, S., Moritz, R. L., Carver, J. J., Wang, M., Ishihama, Y., Bandeira, N., Hermjakob, H., and Vizcaíno, J. A. (10, 2016) The ProteomeXchange consortium in 2017: supporting the cultural change in proteomics public data deposition. Nucleic Acids Research, 45(D1), D1100-D1106.

9. Ng, K.-L. and Taguchi, Y.-H. (September, 2020) Identification of miRNA signatures for kidney renal clear cell carcinoma using the tensordecomposition method. Scientific Reports, 10(1).

10. Tibshirani, R. (1996) Regression Shrinkage and Selection via the Lasso. Journal of the Royal Statistical Society. Series B (Methodological), 58(1), 267-288.

11. Kuleshov, M. V., Jones, M. R., Rouillard, A. D., Fernandez, N. F., Duan, Q., Wang, Z., Koplev, S., Jenkins, S. L., Jagodnik, K. M., Lachmann, A., McDermott, M. G., Monteiro, C. D., Gundersen, G. W., and Ma'ayan, A. $(05,2016)$ Enrichr: a comprehensive gene set enrichment analysis web server 2016 update. Nucleic Acids Research, 44(W1), W90-W97.

12. Huang, D. W., Sherman, B. T., and Lempicki, R. A. (December, 2008) Systematic and integrative analysis of large gene lists using DAVID bioinformatics resources. Nature Protocols, 4(1), 44-57.

13. Li, X., Yu, X., He, Y., Meng, Y., Liang, J., Huang, L., Du, H., Wang, X., and Liu, W. (December, 2018) Integrated Analysis of MicroRNA (miRNA) and mRNA Profiles Reveals Reduced Correlation between MicroRNA and Target Gene in Cancer. BioMed Research International, 2018, 1-15.

14. Hong, L., Li, X., Zhou, D., Geng, J., and Chen, L. (March, 2018) Role of Hippo signaling in regulating immunity. Cellular \& Molecular Immunology, 15(12), 1003-1009.

15. Khalili, M., Lombardero, M., Chung, R. T., Terrault, N. A., Ghany, M. G., Kim, W. R., Lau, D., Lisker-Melman, M., Sanyal, A., Lok, A. S., and for the HBRN (2015) Diabetes and prediabetes in patients with hepatitis B residing in North America. Hepatology, 62(5), 1364-1374.

16. Song, C., Lv, J., Liu, Y., Chen, J. G., Ge, Z., Zhu, J., Dai, J., Du, L.-B., Yu, C., Guo, Y., Bian, Z., Yang, L., Chen, Y., Chen, Z., Liu, J., Jiang, J., Zhu, L., Zhai, X., Jiang, Y., Ma, H., Jin, G., Shen, H., Li, L., Hu, Z., and for the China Kadoorie Biobank Collaborative Group (06, 2019) Associations Between Hepatitis B Virus Infection and Risk of All Cancer Types. JAMA Network Open, 2(6), e195718-e195718.

17. Pakpoor, J., Noyce, A., Goldacre, R., Selkihova, M., Mullin, S., Schrag, A., Lees, A., and Goldacre, M. (2017) Viral hepatitis and Parkinson disease. Neurology, 88(17), 1630-1633.

18. Wang, Y.-R., Wang, Q.-H., Zhang, T., Liu, Y.-H., Yao, X.-Q., Zeng, F., Li, J., Zhou, F.-Y., Wang, L., Yan, J.-C., Zhou, H.-D., and Wang, Y.-J. (March, 2016) Associations Between Hepatic Functions and Plasma AmyloidBeta Levels-Implications for the Capacity of Liver in Peripheral Amyloid-Beta Clearance. Molecular Neurobiology, 54(3), 2338-2344.

19. Ji, M., xiu Xie, X., qun Liu, D., lin Yu, X., Zhang, Y., Zhang, L.-X., wei Wang, S., ru Huang, Y., and tian Liu, R. (June, 2018) Hepatitis B core VLP-based mis-disordered tau vaccine elicits strong immune response and alleviates cognitive deficits and neuropathology progression in Tau.P301S mouse model of Alzheimer's disease and frontotemporal dementia. Alzheimer's Research \& Therapy, 10(1). 
10 Nucleic Acids Research, YYYY, Vol. $x x$, No. $x x$

20. Ritchie, M. E., Phipson, B., Wu, D., Hu, Y., Law, C. W., Shi, W., and Smyth, G. K. (2015) limma powers differential expression analyses for RNA-sequencing and microarray studies. Nucleic Acids Research, 43(7), e47.

21. Tusher, V. G., Tibshirani, R., and Chu, G. (April, 2001) Significance analysis of microarrays applied to the ionizing radiation response. Proceedings of the National Academy of Sciences, 98(9), 5116-5121.

22. Li, J., Lu, Q., and Wen, Y. (11, 2019) Multi-kernel linear mixed model with adaptive lasso for prediction analysis on high-dimensional multiomics data. Bioinformatics, 36(6), 1785-1794.

23. Lun, A. T. and Smyth, G. K. $(11,2015)$ csaw: a Bioconductor package for differential binding analysis of ChIP-seq data using sliding windows. Nucleic Acids Research, 44(5), e45-e45.

24. Argelaguet, R., Velten, B., Arnol, D., Dietrich, S., Zenz, T., Marioni, J. C., Buettner, F., Huber, W., and Stegle, O. (2018) Multi-Omics Factor Analysis-a framework for unsupervised integration of multi-omics data sets. Molecular Systems Biology, 14(6), e8124.

25. Singh, A., Shannon, C. P., Gautier, B., Rohart, F., Vacher, M., Tebbutt, S. J., and Cao, K.-A. L. (January, 2019) DIABLO: an integrative approach for identifying key molecular drivers from multi-omics assays. Bioinformatics, 35(17), 3055-3062.

26. Seoane, J. A., Day, I. N. M., Gaunt, T. R., and Campbell, C. (October, 2013) A pathway-based data integration framework for prediction of disease progression. Bioinformatics, 30(6), 838-845.

27. Jacobson, R. M., Swan, A., Adegbenro, A., Ludington, S. L., Wollan, P. C., and Poland, G. A. (2001) Making vaccines more acceptable methods to prevent and minimize pain and other common adverse events associated with vaccines. Vaccine, 19(17), 2418-2427. 